\title{
SmarTS Lab - A Laboratory for Developing Applications for WAMPAC Systems
}

\author{
L. Vanfretti, M. Chenine, M. S. Almas, R. Leelaruji, L. Ängquist, and L. Nordström
}

\begin{abstract}
At the core of the development of "Smart Transmission Grids" is the design, implementation, and testing of synchronized phasor measurement data applications that can supplement Wide-Area Monitoring, Protection, and Control Systems (WAMPAC). Nevertheless, the development of new PMU data-based WAMPAC applications has been relatively slow. The great potential of WAMPAC systems is being limited by this, and efforts are needed so that new applications can be developed. The slow rate of development of these applications is strongly related to, among other factors, the application development approach used. This article starts by discussing the needs and approaches for developing WAMPAC applications that exploit synchronized phasor measurements, and illustrates how one of these approaches has been achieved. A preliminary work carried out to develop and implement a Smart Transmission System Laboratory (SmarTS Lab), a hardware and softwarebased system for developing and analyzing "Smart Transmission Grids" paradigms and applications for WAMPAC systems, are described. The laboratory's conceptual architecture and hardware and software implementation are presented, and some of its components are described. Finally, the article illustrates proofof-concept examples of how PMU data-based applications can be developed.
\end{abstract}

Index Terms-Synchronized Phasor Measurements, PMU, Wide-Area Monitoring Systems, Wide-Area Control Systems, Wide-Area Protection Systems, Wide-Area Monitoring Protection and Control Systems, Smart Transmission Grids, Real-Time Simulation, SmarTS Lab

\section{INTRODUCTION}

$\mathbf{T}$ He development and implementation of Wide-Area Monitoring, Protection and Control Systems (WAMPAC) [1] exploiting synchronized phasor measurement data for providing coherent real-time data for enhancing power system reliability has seen a significant increase in the last few years [2]. In the Nordic region there have been continued efforts for the development of Wide-Area Monitoring Systems (WAMS) [3] for enhanced monitoring [4], and more recently, Wide-Area Control Systems for improving power system damping [5]. At the heart of WAMS are phasor measurement units (PMU), the measurement device of choice, and their supporting infrastructure which is formed by communication networks

Manuscript submitted to the IEEE PES General Meeting 2012.

L. Vanfretti, M. S. Almas and R. Leelaruji are with the Electric Power Systems Division, School of Electrical Engineering, Royal Institute of Technology (KTH), Teknikringen 33, SE-100 44, Stockholm, Sweden. E-mail: luigiv@kth.se,msalmas@kth.se, rujiroj@kth.se.

L. Vanfretti is supported by the STandUP for Energy collaboration initiative and the KTH School of Electrical Engineering.

M. Chenine and L. Nordström are with the Industrial Information \& Control Systems Division, School of Electrical Engineering, KTH Royal Institute of Technology, SE-100 44, Stockholm, Sweden. E-mail: moustafac@ics.kth.se, larsn@ics.kth.se

L. Ängquist is with the Electrical Energy Conversion Division, School of Electrical Engineering, Royal Institute of Technology (KTH), Teknikringen 33, SE-100 44, Stockholm, Sweden. E-mail:lennart.angquist@ee.kth.se. and computer systems capable of handling PMU data and other information (usually called Phasor Data Concentrators (PDCs)) [6]. WACS are more complex systems which include the implementation of closed-loop wide-area controllers capable of providing reactive power compensation for mitigating power system transient stability, power system oscillations, and voltage collapse [5], [7]. Wide-Area Protection Systems (WAPS), on the other hand, involve intelligent tripping within Remedial Action Schemes (RAS) for different aims including optimized load shedding [8].

At the core of the development of "Smart Transmission Grids" [9] is the design, implementation, and testing of synchronized phasor measurement data applications that can supplement WAMPAC systems. Nevertheless, the development of PMU data-based WAMPAC applications has been relatively slow [9] in comparison with the increased interest and deployment of WAMS around the world [2]. The great potential of WAMPAC systems is being limited by this, and efforts are needed so that new applications can be developed and integrated in these systems for enhanced monitoring, protection and control of electrical power networks. The slow rate of development of these applications is strongly related to, among other factors, the application development approach used. There are different approaches for developing PMU data-based applications, these include: (i) the use of archived data [10], (ii) coupling of proprietary off-line power system simulation software with communication networks and software-intensive systems simulators [11]-[14], and to be discussed in this paper, a relatively less explored approach (iii) the use of realtime hardware-in-the-loop simulators interfaced with physical devices and ad hoc prototype WAMPAC systems [15]-[17].

This article describes a preliminary work carried out to develop and implement a Smart Transmission System Laboratory (SmarTS Lab), a hardware and software-based system for developing and analyzing "Smart Transmission Grids" paradigms and applications for WAMPAC systems. The laboratory's conceptual architecture and hardware and software implementation are presented, and some of its components are described. We illustrate the feasibility of this approach with proof-of-concept examples of how PMU data-based applications can be developed. The applications developed so far are only prototypes and not production level software, however, the intention here is to show the potential and limitations of the development approach enabled by the SmarTS Lab facility.

The reminder of this article discusses the following topics. In Section II the needs and approaches for developing PMUBased WAMPAC applications are reviewed and justify the implementation of the SmarTS Lab; this section also offers an outlook on the difficulties involved in performing simulations of hybrid systems comprised by power systems and software- 
Opal-RT

Real-Time Simulator

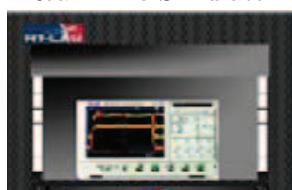

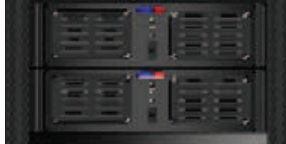

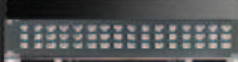

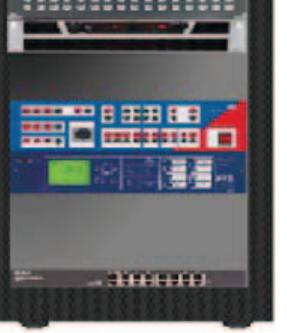

(a) Conceptual Architecture of SmarTS Lab. Measurement and data streams are indicated, non-exclusively, as follows: blue for WAMS, red for WAPS, and green for WACS applications. Solid lines indicate measurement streams, while dotted lines indicate digital data streams over IP.

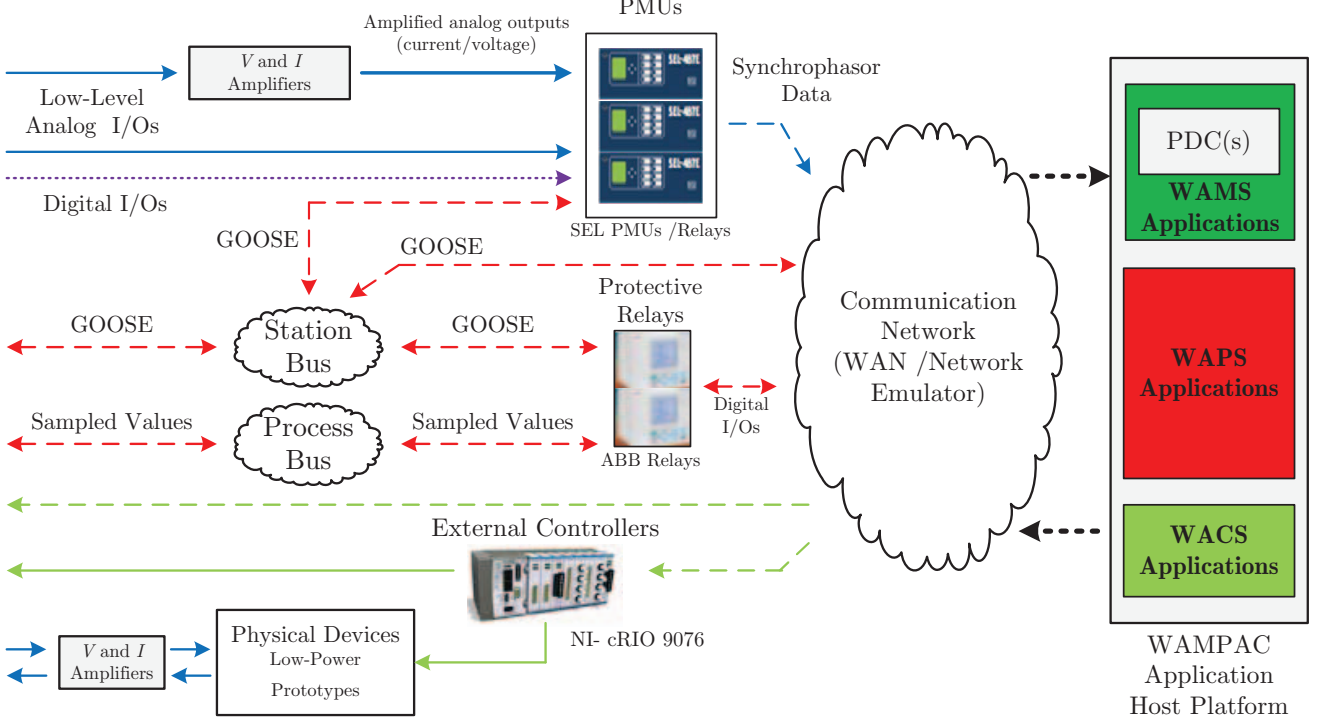

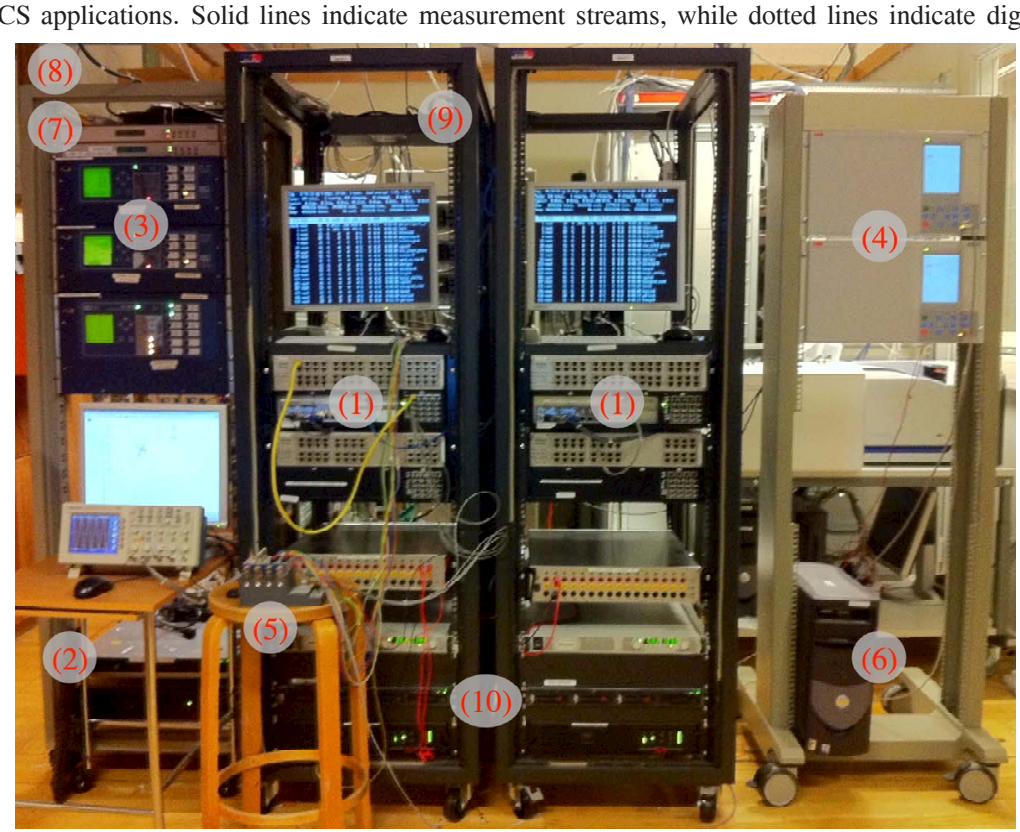

(b) Hardware Implementation of SmarTS Lab as of Dec. 2011.

Fig. 1. Conceptual Architecture and Implementation of SmarTS Lab as of Dec. 2011.

intensive and communication-intensive systems. Section III introduces SmarTS Lab by describing its conceptual architecture, hardware and software implementation, and some of its major components as of December 2011. In Section IV, proofof-concept examples of how PMU data-based applications can be developed are illustrated. Finally, in Section V, conclusions are drawn and future work is briefly outlined.

\section{Needs And Current Approaches for Developing PMU-BASED WAMPAC APPLICATIONS}

\section{A. Need for Development of PMU-Based Applications}

Synchronized phasor measurement data applications, and their implementation within WAMPAC systems are seen as the main building blocks to achieve the miracle of "Smart
Grids" at the transmission systems level. Although there have been important efforts for development PMU-data based applications in North America [18], it has been recognized that the great potential of WAMPAC systems to provide real-time data with synchronized and accurate time-stamping is being limited by the slow rate of inception of new applications [9] that can effectively harvest data and use it for monitoring, protection, and control purposes. There are many factors limiting the development of these applications, one of these aspects is that the necessary supporting infrastructure required to collect, process and transport coherent real-time data that can meet application requirements needs to be developed to support a different number of applications [19]. On the other hand, the determination of specific application requirements is difficult to determine for applications that have not even been envi- 
sioned [9], [19]. From these facts, it becomes apparent that to increase the rate of inception of new WAMPAC applications, it is necessary to set up an approach that encompasses a data infrastructure which can provide real-time data to WAMPAC applications, the power system as such, and the measurement, protection, and control devices serving as actuators.

\section{B. Approaches for Developing PMU-Based Applications}

There are different alternatives for developing new applications using synchrophasor data which can be used in WAMPAC systems. Firstly, PMU data can be harvested from PMUs installed at transmission or distribution networks through ad hoc WAMS systems [6]. For transmission-level data, this has a drawback that researchers need to secure nondisclosure-agreements, and be provided with other SCADA information [10]. A second drawback of this approach is that the applications developed will be limited to off-line analysis methodologies, simply by the nature of the data, and only the post mortem aspects of WACS and WAPS can be analyzed. Hence, on-line applications for real-time monitoring are difficult to develop under this approach, and those for WACS and WAPS are not possible to develop.

In order to develop applications that intrinsically account for power system dynamics, software-intensive and communication-intensive systems, and their interaction, one approach that has been attempted by other researchers is the coupling of standard proprietary off-line power system simulation tools with simulators capable of modelling softwareintensive and communication-intensive systems. There are several technical issues regarding the re-use of these two kind of simulators that make this approach difficult [13]. Among them, it is worth to note that proprietary power system simulation software has not been designed to deal with new data being streamed run-time and require a run-time infrastructure [11]. In addition, the exchange of data between the two types of simulators is limited to particular time-instants as the step size used by the solvers cannot be changed arbitrarily [13], and the occurrence of discrete events such as arrival of data to a controller is delayed until the next simulator step which causes difficulties in simulation of digital control systems [20].

As noted by Nutaro [13], this approach has produced unsatisfactory results due to this technical limitations, and the reuse of existing simulators needs substantial changes to the power system simulation software; this has been the case both with EPOCHS [11] and GridSim [12]. The use of Open Source Software based hybrid-simulators, as outlined in [14], may counter these limitations by using numerical methods that combine discrete and continuous system simulation, and the provision of interfaces to the simulator's run-time execution engine for time management and data injection. However, this work is still in its early stages [13], [14].

\section{Development of PMU-Based WAMPAC Applications using a RT-HIL Approach}

A less explored approach for developing PMU data applications for WAMPAC systems [15]-[17], investigated in this paper, is the use of a Real-Time (RT) Hardware-Inthe-Loop (HIL) simulation environment, coupled to physical devices (such as PMUs and IEDs), and an actual WAMPAC system. This article presents a preliminary work that has been focused on developing a Smart Transmission System Laboratory (SmarTS Lab), a hardware and software-based system for developing and analyzing "Smart Transmission Grids" paradigms and applications for WAMPAC systems.

To properly generate primary data for use with PMUs, RT simulator provides high-resolution three-phase data of voltage and current waveforms [15] in analog and digital input/output (I/O) interfaces, computed using very small time-steps and discrete solvers [21]. The provision of special software libraries to account for sub-time-step events limits the negative effects of coupling discrete control systems [22]. With these facilities, PMUs and any other IED's can be interfaced either through signal amplifiers or the low-voltage I/Os of real-time simulators. This intrinsically allows to account for softwareintensive controls and communication networks by interfacing PMUs and IEDs with PDCs and an application host platform through a Local Access Network (LAN) or Wide Area Network (WAM) by streaming data through their Ethernet ports using TPC/IP and the synchrophasor data protocol IEEE C37.118 [23].

Although the communication network is embedded in this approach, it might not be sufficient to properly emulate actual WAN networks or to consider other communication mechanisms. In this case, when a more complex communication network structure needs to be taken into account, it is possible as with other approaches to interface communication network emulators [24], [25] by routing data streams to a larger emulated communication network model. This approach, while less explored, has a great potential for the development of RT applications involving real-time deterministic computers that can be used to implement wide-area controllers [7], in particular for wide-area power oscillation damping [5], and the coordination of real-time controllers with protective relays [26].

\section{ARChitectural And COMPONENTS OF THE SMARTTS LABORATORY}

\section{A. Conceptual Architecture}

The purpose of SmarTS Lab is to develop different wide area measurement, protection and control applications which exploit PMU data, that when functional, can be utilized by transmission system operators and utilities for safe, efficient and reliable operation of power systems. This includes design and validation of real-time applications involving deterministic computers that can be used to implement wide-area controllers [7], in particular for wide-area power oscillation damping [5], and the coordination of real-time controllers with protective relays [26], which requires the implementation of station and process buses for data exchange. With such aim it becomes necessary to include in the architecture design and implementation, the use of actual physical devices, and ad hoc communication networks and application development hosts. This section describes the conceptual architecture, implementation, and major components used in the laboratory. 
TABLE I

RT Simulator MAin FEATURES

\begin{tabular}{ll}
\hline Features & Description \\
\hline Number of Cores & 2 HIL boxes each with 12 Inter i7 3.3 GHz cores \\
\hline Analog Inputs & $32(+-10 \mathrm{~mA}$ and $+-100 \mathrm{~V})$ \\
\hline Analog Outputs & $128(+-10 \mathrm{~mA}$ and $+-16 \mathrm{~V})$ \\
\hline Digital Inputs & $128(+-4 \mathrm{~V}$ to $+-30 \mathrm{~V}$ and $6 \mathrm{~mA})$ \\
\hline Digital Outputs & $128(+-4 \mathrm{~V}$ t0 $+-30 \mathrm{~V}$, sink up to $100 \mathrm{~mA}$ current $)$ \\
\hline High Voltage DC & 16 Digital outputs $(250 \mathrm{~V}$ DC and up to .125 A current) \\
\hline Interface & IEC 61850-8-1 (GOOSE) and IEC 61850-9-2 Sampled \\
\hline Communication & Values through two separate Ethernet ports. \\
\hline
\end{tabular}

Figure 1 depicts the conceptual architecture of SmarTS Lab. Measurement and data streams are indicated, non-exclusively, as follows: blue for WAMS, red for WAPS, and green for WACS applications. Solid lines indicate measurement streams, while dotted lines indicate digital data streams over IP. An RT simulator is used to emulate the power system and to interface with physical devices. Data for WAMS applications is streamed from PMUs into an ad hoc communication network, where it is sent to a WAMPAC application host platform which includes PDCs. WACS applications stream data either directly to the simulator over IP or to external controllers that can drive models in the RT simulator or low-powered physical devices which are interfaced through the I/Os of the simulator. As for WAPS applications, the simulator streams data using IEC 61850 , using sampled values to communicate with the process bus or GOOSE for the Station Bus, these in turn communicate with the protective relays and the WAPS applications.

\section{B. Hardware and Software Implementation}

The SmarTS-Lab currently deployed at KTH is comprised of several components. These include hardware components and software components, which are different PDCs and application host platforms that allow users to develop applications for wide area measurement based on the data acquired from these hardware components.

The current laboratory set-up consists of both software and hardware components, as shown in Figure 1b, where the current implementation as of December 2011 is shown ${ }^{1}$. (1) The power system is simulated using the eMegaSim RealTime Simulator from Opal-RT [27], capable of providing realtime analog and digital I/Os for its interfacing with hardware components. (2) The WAMPAC application host platform includes the PDC and takes the form of either proprietary software solutions from Schweitzer Engineering Laboratories (SEL) and/or solutions built in-house [28] using Open Source Software [29] and other libraries, as briefly discussed below.

The WAMPAC application host platform interfaces with the RT simulator through the following hardware components: (3) protection relays with embedded PMU functions from Schweitzer Engineering Laboratories (SEL) [30], (4) line differential protection relays from ABB with Optical Ethernet Module (OEM) for station and process bus implementa-

\footnotetext{
${ }^{1}$ Refer to Fig.1b in the following description to map to the numbers listed to the photograph.
}

tion [31], current and voltage amplifiers from Megger [32] (not shown), (5) a compact Reconfigurable Input/Output (cRIO) real-time controller from National Instruments, (6) a PC with a communication networks emulator (Modelnet [24]) and (7) a GPS substation clock from Arbiter Model 1094B [33] fed by (8) a GPS antenna which provides time stamping to the PMUs and IEDs.

The IED's stream data over TCP/IP using a (9)network switch, which also allows users to transfer models to the realtime targets from four independent workstations (not shown). (10)Two servers allow access to the real-time simulator from other locations within KTH. There are additional devices that provide ancillary services for the facility which are not listed here.

\section{Real Time Simulator}

The RT simulators help to model power systems and then simulate them, with very high resolution and small time-steps, to accurately emulate the behaviour of actual power systems. Here, the eMEGAsim real time digital simulator [27] was procured from Opal-RT and deployed in the lab. The major features of the simulator are shown in Table I. With this configuration, the RT simulator allows for the simulation of large power grids while including detailed models of HVDC, FACTS, wind farms, etc., and hardware-in-the-loop interfacing of physical devices.

\section{Physical Devices and their Interface with the RT Simulator}

The overall inter-connection of the Opal-RT simulator with the PMU/relays and vendor softwares is shown in Fig. 2. The analog outputs of the simulator are low level voltage and current signals. These analog outputs of the simulator are connected to the current and voltage inputs of the amplifiers procured from Megger. The amplifiers step up the low level signals to the level acceptable to be fed to the protection relays and PMUs (i.e. $100 \mathrm{~V}$ and $1 \mathrm{~A}$ ). These amplified three-phase voltage and current are directly fed to the current transformer (CT) and voltage transformer (VT) inputs of the PMUs and protection relays from SEL (SEL-421 distance relay and SEL487E transformer differential protection relay), ABB (two RED-670 Line Differential Relays) and Arbiter (Model 1133A Power Sentinel).

Vendor specific softwares such as SEL's AcSELerator Quickset, ABB's PCM 600 and Arbiter's Power Sentinel are used to configure the PMUs and protection relays to update CT and VT ratios, selecting measurements to be included in synchrophasor data streamed out of the PMUs, activating the protection functions (e.g., overcurrent, distance, etc.) and developing a substation architecture based on GOOSE messages.

The modeling platform for Opal-RT real time simulator is MATLAB/ Simulink SimPowerSystems which can be modified using Opal-RT library. The software interface of the OpalRT simulator known as RT-Lab allows the users to build, compile and execute the MATLAB/Simulink SimPowerSystemi $i \frac{1}{2}$ s model in real time. This overall work-flow to obtain data streams from building a model to receiving real-time data is shown also in Fig. 2. 


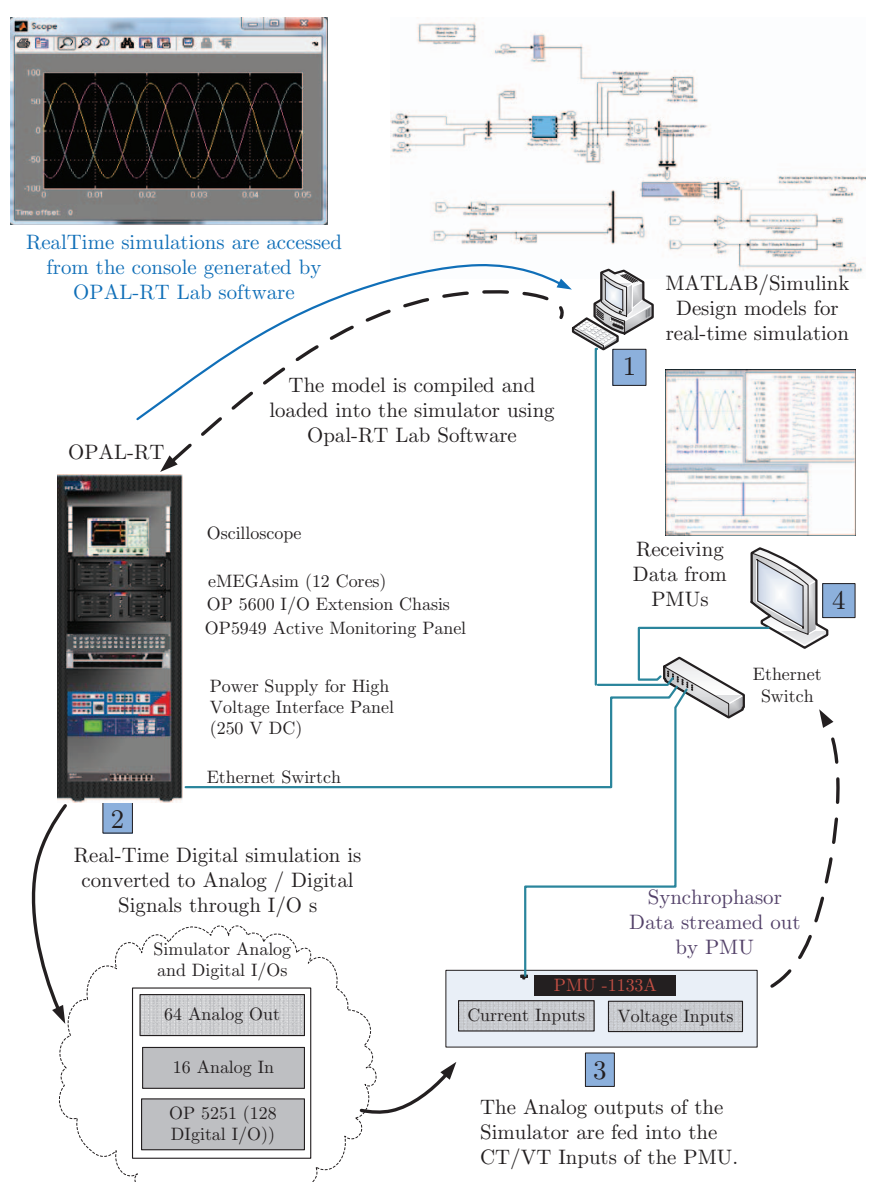

Fig. 2. Model-to-Data Work-Flow for Generating Real-Time Data Streams

Real-time deterministic computers (cRIO 9076) from National Instrument (NI) allow the flexibility to program FieldProgrammable Gate Array (FPGA) which is ideal for embedded control and monitoring applications, specially for advanced control algorithms and high-speed analog and digital $\mathrm{I} / \mathrm{O}$. Currently, the FPGA is programmed to operate as a PMU and its current and voltage modules are directly fed from the analog outputs of Opal-RT real time simulator. The synchronized phasor outputs from the NI's PMU are received in a LabView application in one of the workstations in the laboratory. There is also a web-based configuration and diagnostics interface for the NI's PMU. In the future, these NI equipments will be used to develop models for fast controllers of FACTS and HVDC systems.

\section{E. WAMPAC Application Host Platforms}

1) Use of Commercial Proprietary Solutions: For teaching and learning purposes, it is helpful to use commercial proprietary solutions when deploying a WAMS Application Host Platform. To this end, one of the realizations of the application host platform has been implemented using solutions from SEL. However, there are several limitations to develop new WAMPAC applications in this realization, which will not be listed here.

The synchrophasor data streamed out from the IEDs is sent through the Ethernet channel and received on a server

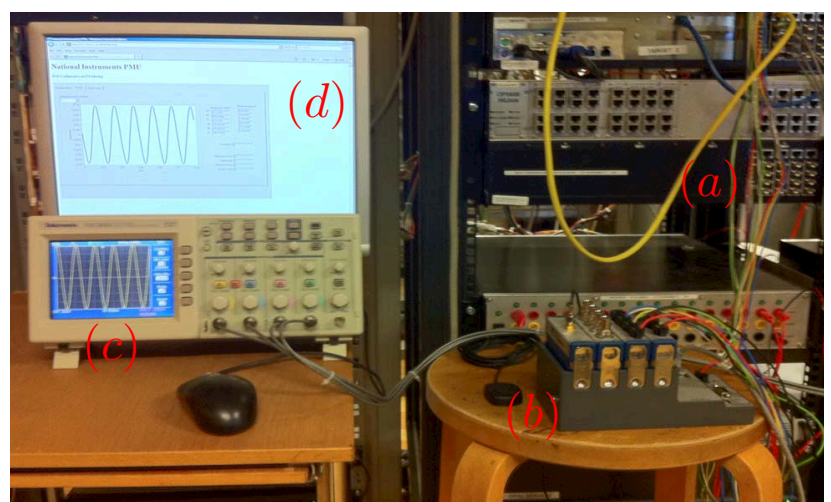

Fig. 3. NI cRIO 9076 RT Deterministic Computer Programmed to Operate as a PMU interfaced with the RT simulator. (a) RT-Simulator, (b) cRIO 9076 and GPS Antenna, (c) oscilloscope showing the RT-Simulator output for phase A of the Voltage Magnitude measured from the simulator's analog I/Os (d) LabView visualization application showing also phase A of the Voltage Magnitude sent through TCP/IP.

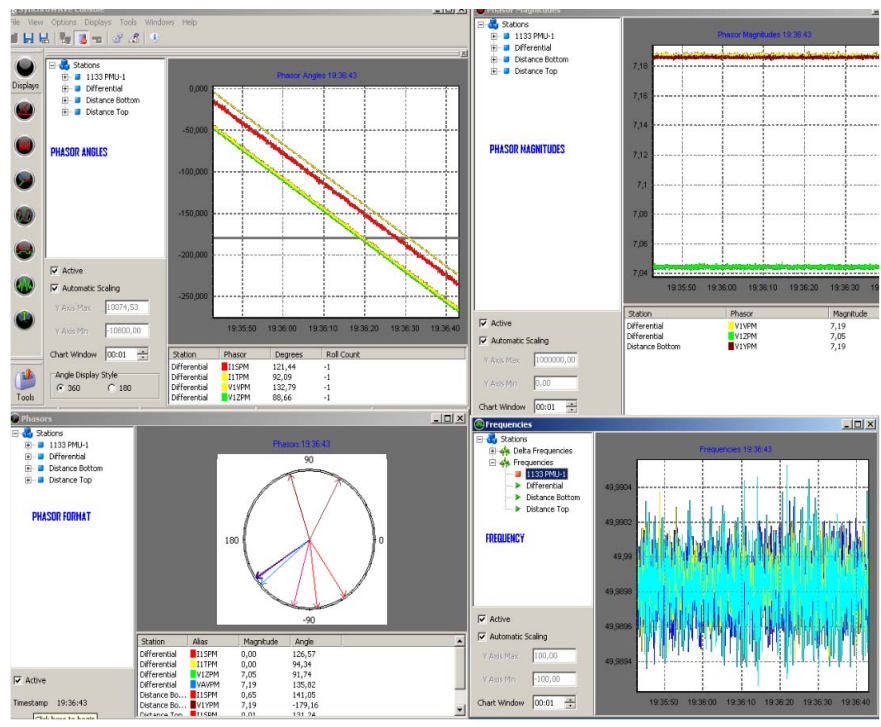

Fig. 4. Synchrophasor Data Graphical Representation using SEL's Synchrowave Console

where three softwares are installed, namely: SEL Synchrowave Console which is used for monitoring synchrophasor data in graphical format, SEL Synchrowave Server which is a Phasor Data Concentrator that time-aligns the synchrophasor input data from different PMUs and broadcast it in a single stream of synchrophasor output. On the other hand, SEL Synchrowave Archiver is implemented to store the synchrophasor data directly or by a trigger in the form of CSV or Comtrade file. Figure 4 shows the SEL Synchrowave Console which is processing the sycnhrophasor data streaming out from PMUs and representing them in the graphical format in real-time.

2) openWAMs and PowerIT Platform [28]: The openWAMS platform is an important part of SmartTS Lab, that provides the platform for developing monitoring and control applications. The openWAMS platform consists of several third party open-source components and libraries as well as components developed to integrate them. These include components that sort PMU data and those that exploit the sorted data which are openPDC system and openHistorian [29]. The open PDC and openHistorian provide 


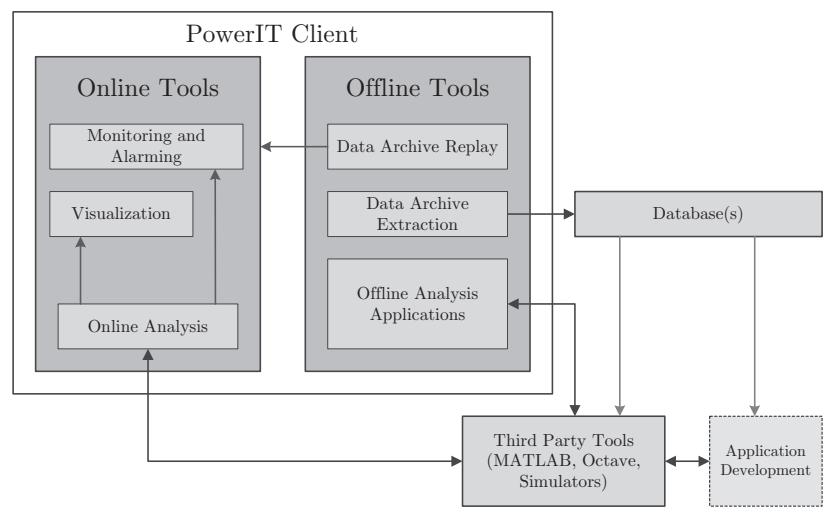

Fig. 5. Functionality of the PowerIT Client System

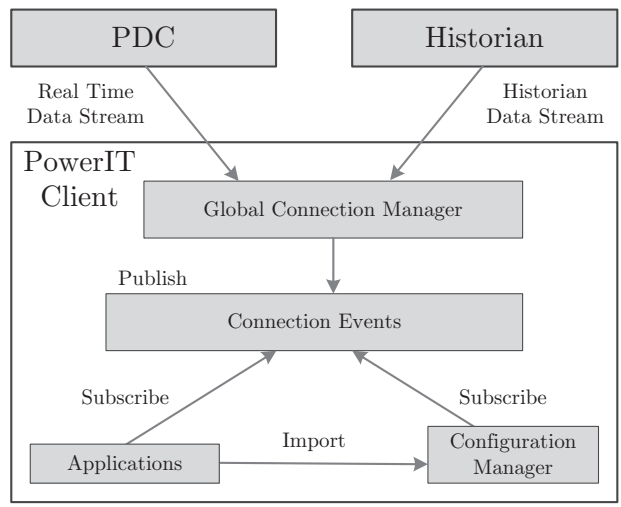

Fig. 6. The main architectural components of the PowerIT client system

functionality for concentrating, archiving and managing phasor data. Coupled with the openWAMS platform is the PowerIT Client system that is the platform's host where applications for on-line and off-line analysis and testing can be deployed.

Figure 5 gives an overview of how functionality is partitioned in the PowerIT client and associated tools. The system has the following application categories: (1) on-line real-time monitoring, (2) on-line real-time event processing, (3) offline replay of data for analysis, and (4) server and client management. This system has been designed to offer a multiuser environment where users have an access to certain system functionality according to authorization such as on-line monitoring, off-line analysis tools, and server configuration. The PowerIT [28] client system makes use of many of the libraries associated with the open PDC, for example the Tennessee Valley Authority (TVA) Code Library [29] and openPDCï $i \frac{1}{2} \mathrm{~s}$ Phasor Protocol Library.

Figure 6 depicts the main architectural components of the PowerIT client system. This architecture is reconfigurable to several protocols which results to flexibly connect to either a PDC server, measurement historian and/or a remote device. The applications in the system are designed to plug-in to the global connection object and subscribe to events fired by that object. A global connection object event can be for example a new measurement from the server that has been received, in which case, the applications respond by utilizing this measurement according to its task. Applications also import a global configuration manager. The global configuration manager simplifies the management of various configurations related to connection between the client system and the PDC. In fact, the configuration manager also subscribes to the events of the global connection object but only to those events relating to configuration changes. By importing the configurations manager, application can dynamically respond to changes in the system configuration. See more details in [28].

Off-line application development refers to the use of archived data for developing new analysis applications that can be used either in on-line and off-line mode. The PowerIT client systems are also designed to offer tools to support offline analysis and data management to extract archive data for viewing directly in the application or for processing inside other application systems, i.e. MATLAB. Availability of data for forensic analysis is of importance and it often allows the determination of factors involved in disruptive events [34]. PowerIT currently supports data extraction from the historian only in Comma Separated Values. The default format of data extracted with the "Data Extraction" adaptor, is as follows:

$\{0: I\} ;\{1: T\} ;\{2: V\} ;\{3: Q\}$

where $I$ is an internal historian identifier for the signal, $T$ is a time-stamp, $V$ is the value of the signal at the instant given by $T$, and $Q$ is a data quality indicator. In this case the delimiter "," separates the identifier, time stamp and value.

\section{Proof of Concept Examples of Developed PMU DATA APPLICATIONS}

Using the infrastructure and components described in the previous sections, several applications were built. These applications as mentioned earlier, are generally categorized as online or off-line applications. On-line application process fast moving data as they arrive to the client system, measurement by measurement. In contrast, off-line applications process data that is archived and stored, and this is usually done on a batch volume of data. Below, some on-line monitoring applications that have been developed and implemented are described as proof-of-concept examples of the application development approach carried out with the SmarTS Lab facility.

\section{A. A Simple System Overview Monitoring Application}

The simple system overview application in the PowerIT client provides a simplified overview of the system. This includes a Map that displays the approximate location of the PMUs and if they are connected (on-line) or disconnected (off-line). The overview screen also provides two plots to summarize data from on-line PMUs; these plots average system frequency and phasor angles. Furthermore, the systems have a traffic light indicator to provide a simplified state of indication of the state of the system.

\section{B. A Simple Average Frequency Monitoring Application}

The average frequency monitoring application in the PowerIT client calculates the average frequency given a set of frequency values from several PMUs. The application is an adaption of the Frequency Monitoring function of the Power Calculations Adapter that is bundled with openPDC [29]. This 


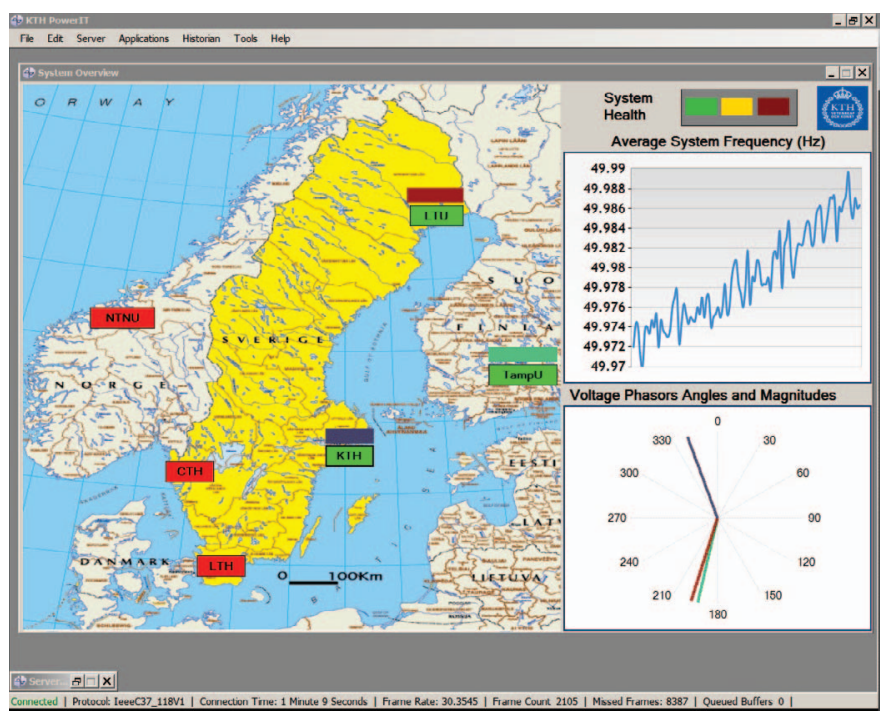

Fig. 7. KTH PowerIT Overview Application

application calculates the average frequency as each value arrives at the client system; this is done simply by adding each frequency value to the a "Frequency Total" variable and then dividing by the number of frequency values received. The application also allows plotting the frequency of each PMU on the same graph as the average frequency trend. Figure 8 shows the average frequency calculated from two PMUs from SEL namely the differential protection relay SEL $487 E$ and distance protection relay SEL 421. These two PMU are hard-wired to the simulator.

\section{On-line Electromechanical Mode Estimation}

The on-line mode estimation application is based on work outlined in [34] where archived data is used to estimate electromechanical properties of power systems. The application collects real-time measurements for a pre-specified time window and then processes these measurements in two steps: first preprocessing the data buffer, and then performing a spectral estimation. Figure 9 shows a screen shot of this application.

The processing part of the mode estimation application was originally written in MATLAB. This code was reorganized into more manageable functions and exported using the MATLAB NE Compiler as independent libraries to be used by the mode estimation application component of the PowerIT client system.

\section{CONClusions AND Further Work}

After critically discussing the current approaches for development of PMU-based WAMPAC applications, this article has described the substantial work carried out to develop and implement a laboratory that uses one of the less explored paradigms for application development: the RT-HIL approach. This has amounted to the establishment of the Smart Transmission System Laboratory (SmarTS Lab), a hardware and software-based system for developing and analyzing "Smart Transmission Grids" paradigms and applications for

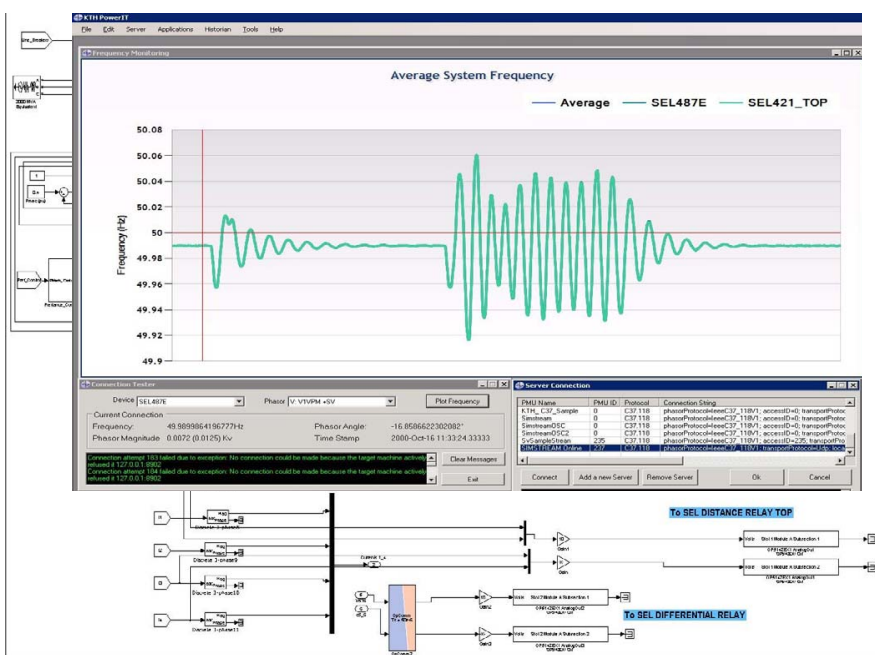

Fig. 8. A screen-shot showing openWAMS and PowerIT receiving data from two PMUs deployed in Hardware-in-the-loop fashion with the Opal-RT

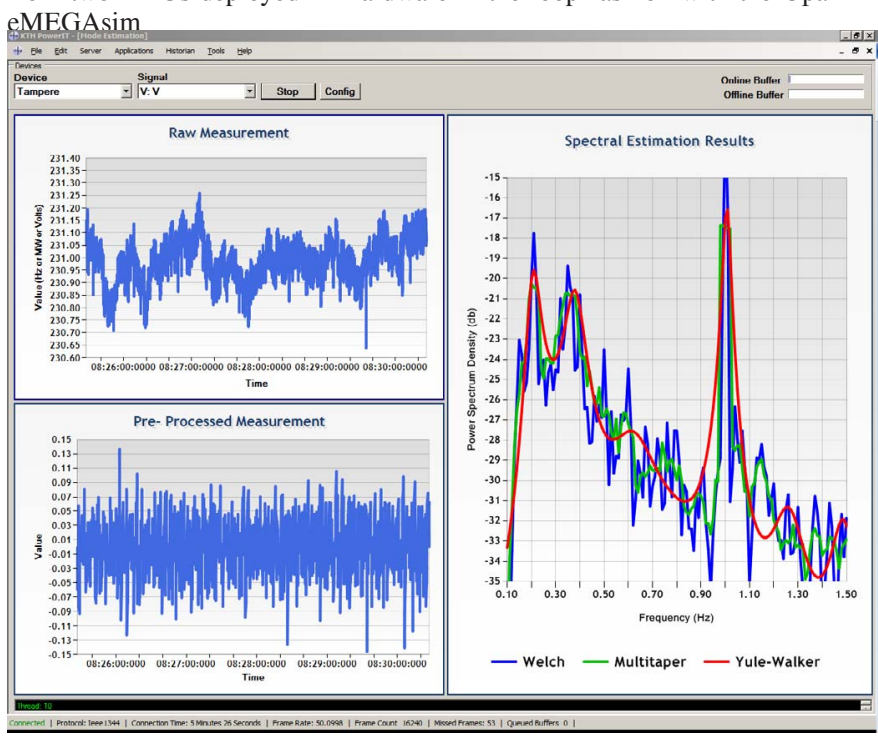

Fig. 9. The on-line Mode Frequency Estimation Display in the PowerIT Client System

WAMPAC systems. The laboratory's conceptual architecture and hardware and software implementation were described and proof-of-concept examples of how PMU data-based applications can be developed were presented.

Of course, as with other research approaches for developing WAMPAC applications, there are limitations to this application development approach. The RT-HIL approach on which SmarTS Lab is constructed has both technical and economical drawbacks. The technical limitations are related to the possible number of outputs that can be used to interface PMU devices, which is limited; and also, limits in small-time step computation due to high number of output signals, power system network size and component model complexity. The economical constraints are only related to cost: for a research laboratory only a limited number of units can be interfaced due to budgeting issues. To overcome these difficulties, "virtualization", i.e. the development of an entirely software-based monitoring, control and protection devices, for use with RTHIL can be an attractive approach to counter these limitations. Such approach has shown promise in terms of proof of concept 
implementation of a PMU [25]; however more work needs to be done in this area.

Regardless of these limitations, the availability of this laboratory is attractive for RT applications involving realtime deterministic computers that can be used to implement wide-area controllers [7], in particular for wide-area power oscillation damping [5], and the coordination of real-time controllers with protective relays [26]; development of such applications is not possible with other simulation platforms.

\section{ACKNOWLEDGEMENTS}

The generosity of Schweitzer Engineering Laboratories, Pullman, WA, USA; ABB Substation Automation Products, Västerås, Sweden; Megger/Programma, Täby, Sweden; and National Instruments, Oslo and Stockholm; for their donation of different hardware and technical support is deeply acknowledged.

The technical support of Mr. Fredrik Höberg, Mr. Peter Lönn and Dr. Alija Cosic, and the financial support of the Electric Power Systems Division at KTH supporting the development of the laboratory is also gratefully acknowledged.

\section{REFERENCES}

[1] V. Terzija, G. Valverde, D. Cai, P. Regulski, V. Madani, J. Fitch, S. Skok, M. Begovic, and A. Phadke, "Wide-Area Monitoring, Protection, and Control of Future Electric Power Networks," Proceedings of the IEEE, vol. 99, no. 1, pp. $80-93$, jan. 2011.

[2] A. Phadke and R. de Moraes, "The Wide World of Wide-area Measurement," IEEE Power and Energy Magazine, vol. 6, no. 5, pp. 52 -65, september-october 2008.

[3] A. Leirbukt, O. Breidablik, J. Gjerde, P. Korba, K. Uhlen, and L. Vormedal, "Deployment of a SCADA integrated wide area monitoring system," in 2008 IEEE/PES Transmission and Distribution Conference and Exposition: Latin America, aug. 2008.

[4] K. Uhlen, L. Warland, J. Gjerde, O. Breidablik, M. Uusitalo, A. Leirbukt, and P. Korba, "Monitoring amplitude, frequency and damping of power system oscillations with PMU measurements," in 2008 IEEE Power and Energy Society General Meeting - Conversion and Delivery of Electrical Energy in the 21st Century., july 2008.

[5] K. Uhlen, L. Vanfretti, J. O. Gjerde, and V. a. Aarstrad, "Wide-Area Power Oscillation Damper Implementation and Testing in the Norwegian Transmission Network," in submitted for publication, IEEE PES General Meeting 2012, july 2012.

[6] K. Martin and J. Carroll, "Phasing in the technology," Power and Energy Magazine, IEEE, vol. 6, no. 5, pp. 24 -33, september-october 2008.

[7] C. Taylor, D. Erickson, K. Martin, R. Wilson, and V. Venkatasubramanian, "WACS-Wide-Area Stability and Voltage Control System: R\&D and Online Demonstration," Proceedings of the IEEE, vol. 93, no. 5, pp. $892-906$, may 2005.

[8] M. Begovic, D. Novosel, D. Karlsson, C. Henville, and G. Michel, "Wide-area protection and emergency control," Proceedings of the IEEE, vol. 93, no. 5, pp. $876-891$, may 2005 .

[9] A. Bose, "Smart Transmission Grid Applications and Their Supporting Infrastructure," Smart Grid, IEEE Transactions on, vol. 1, no. 1, pp. 11 -19 , june 2010 .

[10] L. Vanfretti, V. H. D, L. Nordström, and J. Gjerde, "A smart transmission grid for Europe: Research challenges in developing grid enabling technologies," in IEEE Power and Energy Society General Meeting, july 2011.

[11] K. Hopkinson, X. Wang, R. Giovanini, J. Thorp, K. Birman, and D. Coury, "EPOCHS: a platform for agent-based electric power and communication simulation built from commercial off-the-shelf components," IEEE Transactions on Power Systems, vol. 21, no. 2, pp. 548 558, may 2006.

[12] D. Anderson, Z. Chuanlin, C. Hauser, V. Venkatasubramanian, D. Bakken, and A. Bose, "Power Grid Communications: Integrated Simulation for Designing Smart Grid Applications," in submitted for publication, IEEE Power and energy Magazine for a Special Issue on Smart Grid Communications, May 2011.
[13] J. Nutaro, "Designing power system simulators for the smart grid: Combining controls, communications, and electro-mechanical dynamics," in Power and Energy Society General Meeting, 2011 IEEE, july 2011.

[14] J. Nutaro, P. T. Kuruganti, L. Miller, S. Mullen, and M. Shankar, "Integrated hybrid-simulation of electric power and communications systems," IEEE Power Engineering Society General Meeting, pp. 1-8, June 2007.

[15] T. Rauhala and P. Järventausta, "Testing the Quality of PMU Output Data Based Subsynchronous Damping Analysis in Real-Time Simulation Environment," in International Conference on Power Systems Transients (IPST '07), june 2007.

[16] H. Kang, B. Cvorovic, C. Mycock, D. Tholomier, and R. Mai, "PMU simulation and application for power system stability monitoring," in IEEE/PES Power Systems Conference and Exposition, 2009 (PSCE '09), march 2009.

[17] T. Bi, Y. Zhang, X. Xiao, P. Forsyth, and R. Wierckx, "Large scale power system simulation and PMU testing using a real time digital simulator," in International Power Engineering Conference, 2007 (IPEC 2007), dec. 2007, pp. $383-388$

[18] M. Parashar and J. Mo, "Real Time Dynamics Monitoring System (RTDMS): Phasor Applications for the Control Room," in 42nd Hawaii International Conference on System Sciences, 2009 (HICSS '09), Jan 2009.

[19] D. Bakken, A. Bose, C. Hauser, D. Whitehead, and G. Zweigle, "Smart Generation and Transmission With Coherent, Real-Time Data," Proceedings of the IEEE, vol. 99, no. 6, pp. 928 -951, june 2011.

[20] O. Nayak, S. Santoso, and P. Buchanan, "Power electronics spark new simulation challenges," IEEE Computer Applications in Power, vol. 15, no. 4 , pp. $37-44$, oct 2002.

[21] C. Dufour, J. Mahseredjian, and J. Beélanger, "A Combined State-Space Nodal Method for the Simulation of Power System Transients," IEEE Transactions onPower Delivery, vol. 26, no. 2, pp. 928 -935, april 2011.

[22] S. Abourida and J. Bélanger, "Real-Time Platform for the Control Prototyping and Simulation of Power Electronics and Drives," in Proceedings of the Third International Conference on Modeling, Simulation and Applied Optimization, Jan 2009.

[23] "IEEE Standard for Synchrophasors for Power Systems," IEEE Std C37.118-2005 (Revision of IEEE Std 1344-1995), pp. 1-57, 2006.

[24] K. Yocum, E. Eade, J. Degesys, D. Becker, J. Chase, and A. Vahdat, "Toward scaling network emulation using topology partitioning," in Modeling, Analysis and Simulation of Computer Telecommunications Systems, 2003. MASCOTS 2003. 11th IEEE/ACM International Symposium on, oct. 2003, pp. $242-245$.

[25] A.T. Al-Hammouri et al, "Virtualization of Sychronized Phasor Measurement Units within Real-Time Simulators for Smart Grid Applications," in submitted for publication, IEEE PES General Meeting 2012, july 2012.

[26] R. Leelaruji, L. Vanfretti et al, "Coordination of protection and VSCHVDC systems for mitigating cascading failures," in 2010 International Conference on Power System Technology (POWERCON), oct. 2010.

[27] eMEGAsim PowerGrid Real-Time Digital Hardware in the Loop Simulator - Opal RT, [Online]. Available: http: / /www. opal-rt.com/.

[28] M. Chenine, L. Vanfretti, S. Bengtsson, and L. Nordström, "Implementation of an experimental wide-area monitoring platform for development of synchronized phasor measurement applications," in IEEE Power and Energy Society General Meeting, 2011., july 2011.

[29] “openPDC: The Open Source Phasor Data Concentrator," available online: http://openpdc.codeplex.com/.

[30] "Protection Relays by Schweitzer Engineering Laboratories," Available online: $\mathrm{http} / / / \mathrm{www}$.selinc.com/protection/.

[31] "Line Differential Relay RED-670 by ABB," Available online: http://www.abb.com/relion.

[32] "Current and Voltage Amplifiers by Megger," Available online: http://www.megger.com/cae/story/Index.php?ID=527.

[33] "GPS Substation Clock by Arbiter," Available online: http://www.arbiter.com/.

[34] L. Vanfretti, L. Dosiek, J. W. Pierre, D. Trudnowski, J. H. Chow, R. García-Valle, and U. Aliyu, "Application of ambient analysis techniques for the estimation of electromechanical oscillations from measured pmu data in four different power systems," European Transactions on Electrical Power, vol. 21, no. 4, pp. 1640-1656, 2011. [Online]. Available: http://dx.doi.org/10.1002/etep.507 